\title{
Etude de quelques propriétés de l'écoulement dans une conduite collabable
}

\author{
Study of some properties of flow in collapsible duct
}

\author{
Marc Bonis \\ Département de Génie Biologique, Université de Compiègne, \\ BP 233, 60206 Compiègne Cédex \\ Christian Ribreau \\ Mécanique Expérimentale des Fluides, Bât. 502, 91405 Orsay
}

\section{Introduction}

Lorsqu'une conduite initialement circulaire est soumise à une surpression extérieure, au-delà d'une certaine valeur de la pression appelée pression critique, la configuration circulaire devient instable, et il s'ensuit une déformation qui peut aboutir à un écrasement complet du tube. La pression critique de flambement se rapproche d'autant plus de zéro que l'épaisseur est faible devant le rayon. Dans le cas des conduites en élastomère, dites "collabables" que nous étudions, le comportement demeure élastique, la déformation est réversible et relativement faible pour les pressions positives ou suffisamment négatives, avec des déformations très importantes au voisinage de zéro.

Lorsqu'une telle conduite est le siège d'un écoulement, l'aire d'une section droite varie avec la valeur locale de la pression. Celle-ci étant imposée par les conditions aux limites et la loi de perte d'énergie par frotte. ments visqueux, la forme du tube s'adapte jusqu'à satisfaction de l'équation de continuité. Ainsi, les relations pression-débit dépendent non seulement de la différence de pression entre l'amont et l'aval, comme dans les tubes rigides, mais surtout des pressions transmurales amont et aval qui déterminent la forme de la conduite, donc la valeur des vitesses et la loi de perte de charge par l'intermédiaire de la loi de déformation du tube.

L'initiative de l'étude de ces écoulements est due aux physiologistes qui se sont intéressés au retour veineux. Un historique en a été fait par Brecher [1]. Les premiers travaux sur modeles concernant les relations pressiondébit dans les tubes collabables sont dus à Holt [2] qui étudia la "pompe thoracique". Lors de l'inspiration, la pression intra-thoracique étant négative, les veines de la cage thoracique se dilatent, et produisent une aspiration qui tend à augmenter le débit veineux. Cette augmentation de la vitesse d'écoulement entraîne une diminution de pression dans les vaisseaux et la fermeture des veines juste avant l'entrée dans le thorax. La résistance à l'écoulement s'accroît, limitant ainsi l'augmentation du débit, et la pression capillaire périphérique est maintenue constante.

Un paramètre important est la vitesse de propagation $C$ des ondes de pression. De par la faible rigidité en flexion de ces vaisseaux, $C$ peut atteindre une valeur minimale de l'ordre de $20 \mathrm{~cm} / \mathrm{s}$, et l'écoulement devient critique pour des valeurs assez basses du débit. Griffiths [3] a montré que l'écoulement de l'urine dans l'urètre masculin est gouverné par un col critique où la vitesse du fluide est égale à $C$, et où le débit est limité. Le col est suivi d'un ressaut réalisant la transition du régime supercritique au régime critique.

Toute étude approfondie nécessite donc la détermination de "l'équation d'état" du tube, c'est-à-dire la loi de variation $S\left(p_{i}-p_{e}\right)$ de l'aire d'une section droite en fonction de la pression transmurale $\left(p_{i}\right.$ et $p_{e}$ pressions intérieure et extérieure, $p_{i}-p_{e}=\Delta p$ ), ce qui permet, d'une part d'en déduire $C(\Delta p)$, d'autre part d'exprimer l'équation de conservation et le coefficient de perte de charge $\Lambda(\Delta p)$.

\section{Loi de déformation}

Les vaisseaux anatomiques, ainsi que les tubes de latex utilisés pour nos expériences ne sont pas parfaitement circulaires et peuvent être assimilés à des ellipses d'axes $2 a$ et $2 b(a>b)$ pour $\Delta p=0$. La pression critique de flambement est alors nulle.

\section{Pression transmurale négative}

Lorsque $\Delta p<0$, le tube subit de grandes déformations et les forces de pression sont équilibrées par les 
moments de flexion de la paroi. Le tube s'applatit, les parois opposées viennent en contact pour la pression $\Delta p_{c}$, puis la section devient constituée de deux lobes séparés par un segment de contact rectiligne. Les contraintes dans la paroi demeurent faibles, le périmètre de la section demeure constant et le comportement élastique est linéaire. On peut montrer, à partir des équations générales de l'équilibre d'une coque mince, que le moment $M(s)$ en un point $B$ de coordonnées $x(s)$ et $y(s)$, figure 1 , s'écrit pour $\Delta p>\Delta p_{c}$ :

$$
\begin{array}{r}
M(s)=M(o)-\frac{\Delta p}{2}\left[x^{2}(s)+y^{2}(s)-x^{2}(o)\right]= \\
\frac{E h^{3}}{12\left(1-\nu^{2}\right)}\left[\frac{1}{R(s)}-\frac{1}{R_{0}(s)}\right]
\end{array}
$$

où $h$ est l'épaisseur, $E$ le module de Young, $v$ le coefficient de Poisson. $R_{o}(s)$ et $R(s)$ sont respectivement les rayons de courbure de l'ellipse initiale et de sa déformée aux points homologues d'abcisse $s$.

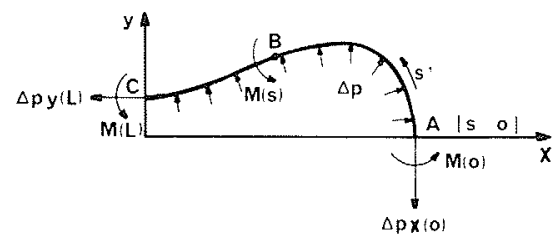

Figure 1 - Equilibre de la section droite du tube pour $\Delta p=p_{i}-p_{e}<0$, les efforts longitudinaux sont négligés.

L'abcisse $x(o)$ et le moment $M(o)$ en $A$, à l'intersection de la paroi avec l'axe $o x$ étant inconnus, l'équilibre de la section est statiquement indéterminé. La relation (1) conduit donc à un système d'équations différentielles non linéaires associé à un problème de conditions aux limites à deux valeurs. Le système a été résolu numériquement à l'intérieur d'un algorithme itératif permettant d'optimiser les valeurs des paramètres surabondants. Deux études expérimentales ont été entreprises pour valider cette solution théorique analogue à celle de Kresh et Noordergraaf [4].

\section{Visualisation de la forme des sections ${ }^{(1)}$ :}

Un échantillon de tube, rempli d'hexanol - 1 pour annuler les effets hydrostatiques, est plongé dans un bain de paraffine en fusion à la température de $95^{\circ} \mathrm{C}$. L'hexanol - 1 n'altère pas les propriétés du caoutchouc, et sa masse volumique $\left(0,82 \mathrm{~g} / \mathrm{cm}^{3}\right)$ est tres voisine de celle de la paraffine à la température de solidification $\left(0,80\right.$ a $0,85 \mathrm{~g} / \mathrm{cm}^{3}$ à $\left.53^{\circ} \mathrm{C}\right)$. Le tube est soumis à une pression transmurale négative, l'ensemble est ensuite lentement refroidi, puis tronçonné. La figure 2 montre la confrontation entre quelques agrandissements photographiques de la section et les résultats du calcul numérique. Cette expérience permet également de vérifier, sur la figure 3 , l'hypothèse d'inextension de la fibre neutre.

(1) Ces expériences ont été réalisées par J. Nahmias au Laboratoire de Mécanique des Fluides d'Orsay.
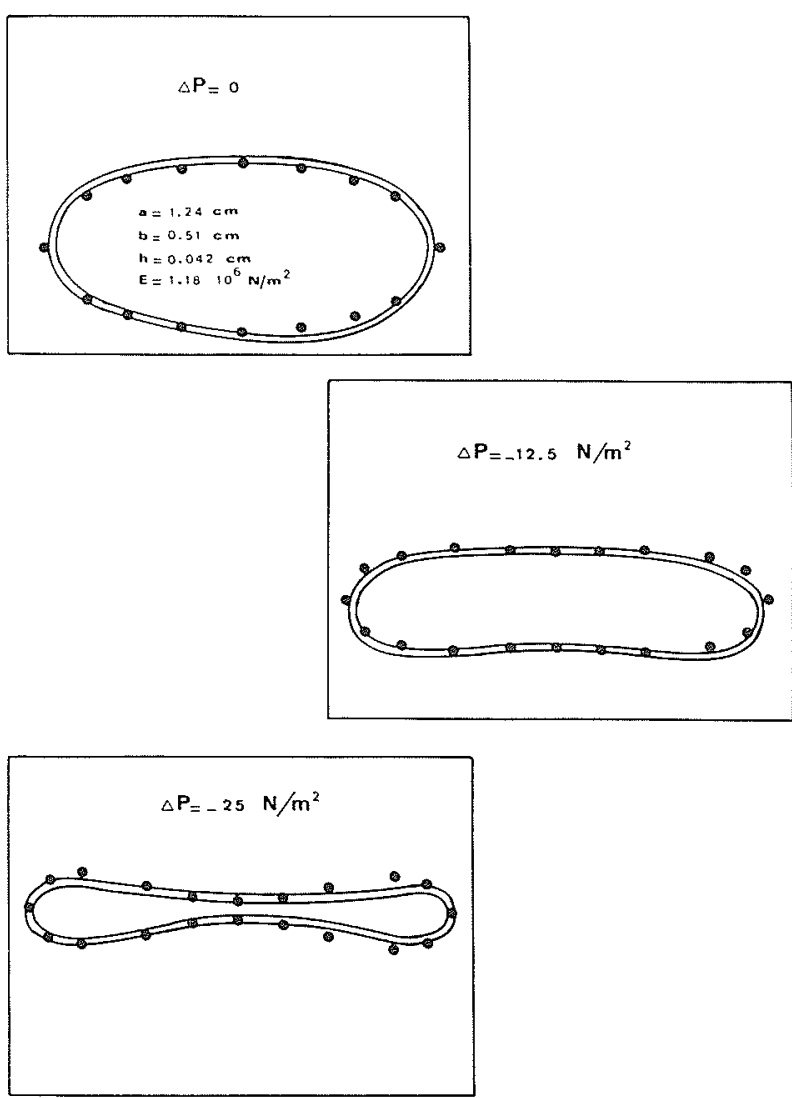

Figure 2 - Formes de la section obtenues à partir des moulages de paraffine pour différentes valeurs de la pression. Comparaison avec les points théoriques solutions de l'équation (1).

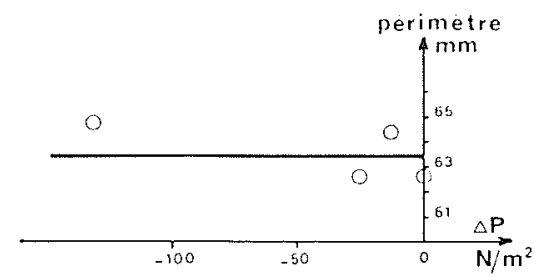

Figure 3 -Vérification de l'hypothèse d'inextensibilité de la paroi lorsque $\Delta p<0$. Le périmètre de la section a été mesuré sur un agrandissement photographique des empreintes de paraffine.

\section{Mesure de $S(\Delta p)$}

Un tube de latex est ligaturé sur deux attaches cylindriques à l'intérieur d'une chambre remplie d'eau. Nous mesurons les variations d'un volume initial connu sous l'effet de la pression. Afin d'éliminer l'influence des effets de bouts, la même expérience est répétée pour deux longueurs d'échantillon ( $1 \mathrm{~m}$ et $0,6 \mathrm{~m}$ ), la courbe $S(\Delta p)$ est ainsi obtenue par différence. Les résultats théoriques et expérimentaux sont présentés sur la figure 4. Pour $2 \Delta p_{c}<\Delta p<\Delta p_{c}$ la solution présentée, bien qu'incorrecte théoriquement, a été utilisée pour son accord avec les résultats expérimentaux. Pour $\Delta p<2 \Delta p_{c}$, l'influence de la configuration initiale 


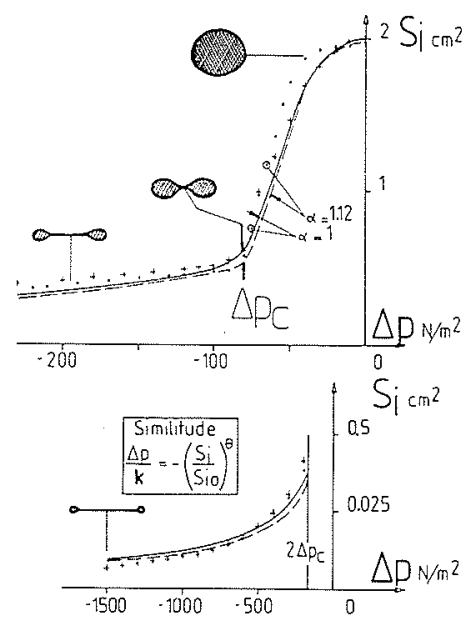

Figure 4 - Aire de la section droite en fonction de la pression pour $\Delta p<0$, pour deux valeurs de l'extension longitudinale : $\alpha=l / l_{o}$. Les parois opposées viennent en contact à la pression $\Delta p_{c}$. Pour $\Delta p<2 \Delta p_{c}$ : loi de similitude.

devient négligeable, et Scherer, Kamm et Shapiro [5], ont trouvé une relation de similitude de la forme :

$$
\frac{\Delta p}{k}=-\left(\frac{S_{i}}{S_{i o}}\right)^{\theta}, \theta=-\frac{3}{2}
$$

que nos résultats viennent confirmer, avec

$$
k=\frac{E h^{3}}{6\left(1-\nu^{2}\right) L^{3}}
$$

où $L$ est le quart du périmètre de l'ellipse. La valeur expérimentale de l'exposant est $\theta=-1,6$.

\section{Pression transmurale positive}

Si $\Delta p>0$, selon la valeur de l'excentricité de l'ellipse, la solution précédente peut être poursuivie pour les pressions faiblement positives. Lorsque la pression est suffisamment grande, la section est circulaire, les forces de pression sont équilibrées par les contraintes tangentielles, et le comportement élastique est en général non linéaire.

Soient $\lambda_{1}, \lambda_{2}, \lambda_{3}$ les coefficients d'extension suivant les axes principaux du tube:

$$
\lambda_{1}=\frac{l}{l_{0}}, \quad \lambda_{2}=\frac{R}{R_{0}}, \quad \lambda_{3}=\frac{h}{h_{0}}
$$

$l_{o}, R_{o}$ et $h_{o}$ : longueur, rayon et épaisseur du tube non contraint (pour $\Delta p=0$ et $\lambda_{1}=1$ ).

Si $\sigma_{1}, \sigma_{2}, \sigma_{3}$, sont les contraintes correspondantes, figure 5, Treloar [6] a montré que pour un tube de caoutchouc soumis à de grandes déformations :

$$
\begin{aligned}
& \sigma_{2}-\sigma_{1}=G\left(\lambda_{2}^{2}-\lambda_{1}^{2}\right) \\
& \sigma_{1}-\sigma_{3}=G\left(\lambda_{1}^{2}-\lambda_{3}^{2}\right) \\
& \text { avec } \quad G=\frac{E}{2(1+\nu)}
\end{aligned}
$$

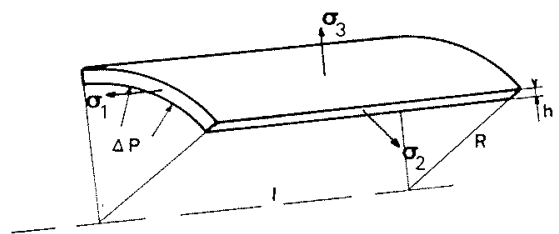

Figure 5 - Contraintes principales $\sigma_{1}, \sigma_{2}, \sigma_{3}$, dans un tube circulaire soumis à une pression $\Delta p$ positive.

Si le matériau est incompressible :

$$
\lambda_{1} \lambda_{2} \lambda_{3}=1
$$

Le tube étant à paroi mince :

$$
\sigma_{3}=0 \quad \text { et } \quad \sigma_{2}=\frac{\Delta p R}{h}
$$

D'après (3), (4), (5), (6), si $\lambda_{1}=\alpha$, on obtient:

$$
\Delta p=\frac{G h_{o}}{R_{o} \alpha^{3}}\left[\alpha^{2}-\left(\frac{S_{o}}{S}\right)^{2}\right]
$$

où $S_{o}$ est l'aire initiale du tube non contraint.

Cette relation est d'autant mieux vérifiée que $\frac{h_{o}}{R_{o}}$ se rapproche de zéro. Afin de tenir compte également de la légère anisotropie des échantillons, nous sommes amenés à définir un coefficient empirique $H$, Ribreau et Bonis [7], tel que

$$
\Delta p=H \frac{h_{o}}{R_{o} \alpha^{3}}\left[\alpha^{2}-\left(\frac{S_{0}}{S}\right)^{2}\right]
$$

Pour les résultats présentés sur la figure $6\left(R_{o}=8,3 \mathrm{~mm}\right.$; $\left.h_{o}=0,45 \mathrm{~mm} ; E=1,31 \times 10^{6} \mathrm{~N} / \mathrm{m}^{2}, \alpha=1,19\right)$, le coefficient $H=4,10 \times 10^{5} \mathrm{~N} / \mathrm{m}^{2}$ est très voisin du terme correctif proposé par Taylor et Gerrard [8] :

$$
H^{\prime}=G\left(1+\frac{h_{o}}{2 R_{o}}\right) /\left(1+\frac{h_{o}}{R_{o}}\right)^{2}
$$

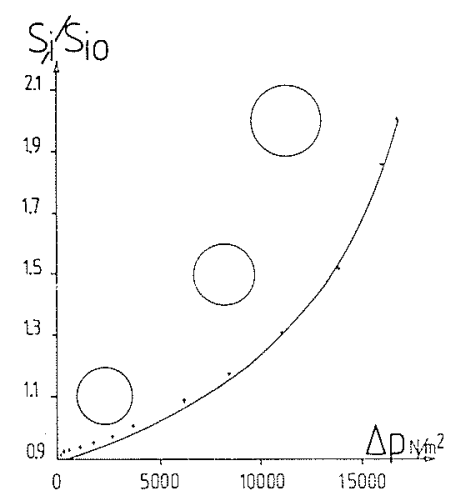

Figure 6 - Aire de la section droite en fonction de la pression pour $\Delta p>0$.

Ces deux types de déformation bien distincts sont séparés par un domaine de raccordement pour lequel nous ne disposons pas actuellement de solution.

L'ensemble des solutions proposées est regroupé sur la figure 7 . 


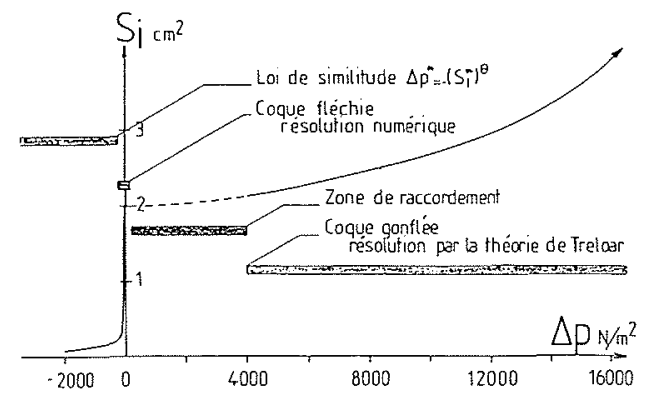

Figure 7 - Différentes solutions théoriques proposées.

\section{Célérité des ondes de pression}

La vitesse de propagation des ondes de pression a été mesurée dans un tube annulaire semi-déformable constitué d'un tube de latex de longueur $4 \mathrm{~m}$ monté à l'intérieur d'un tube en plastique transparent rigide. L'espace annulaire est rempli d'eau, et l'intérieur du tube souple est raccordé à un grand réservoir d'air sous pression. Deux cables d'acier maintiennent les deux tubes sensiblement coaxiaux.

La notion de section collabable est ici étendue à la diminution de l'aire d'une couronne circulaire par gonflement d'un tube distensible. Bien qu'une telle structure soit très éloignée des conditions physiologiques, certains phénomènes fondamentaux peuvent $\mathrm{y}$ être aussi bien mis en évidence, avec l'avantage de disposer d'une loi de déformation analytique, et d'expressions connues pour le coefficient de perte de charge.

Soient $S_{i}$ l'aire intérieure du tube déformable, $S_{e}$ l'aire de l'espace annulaire, et $A_{o}=S_{i}+S_{e}$ l'aire intérieure du tube rigide. On montre aisément, que la célérité des ondes dans une telle conduite annulaire est :

$$
C_{e}^{2}=\frac{S_{e}}{\rho_{e}} \cdot \frac{d \Delta p}{d S_{i}}
$$

\section{Pressions transmurales positives}

On obtient, à partir des équations (8) et (10) :

$$
\begin{aligned}
& C_{e}^{2}=\frac{2}{\rho_{e}} \frac{H h_{o}}{\alpha R_{o}}\left(1-\frac{\Delta p}{H} \frac{R_{o}}{h_{o}} \alpha\right) \\
& {\left[\frac{A_{o}}{S_{i o}} \alpha \sqrt{1-\frac{\Delta p R_{o}}{H h_{o}}} \alpha-1\right] }
\end{aligned}
$$

les résultats théoriques et expérimentaux sont comparés sur la figure 8. L'écart constaté est dû au contact entre le tube de caoutchouc et la paroi rigide, les cables ne pouvant maintenir les deux tubes coaxiaux lorsqu'il y a gonflement. Quand l'espace annulaire devient très petit, la célérité des ondes tend vers zéro.

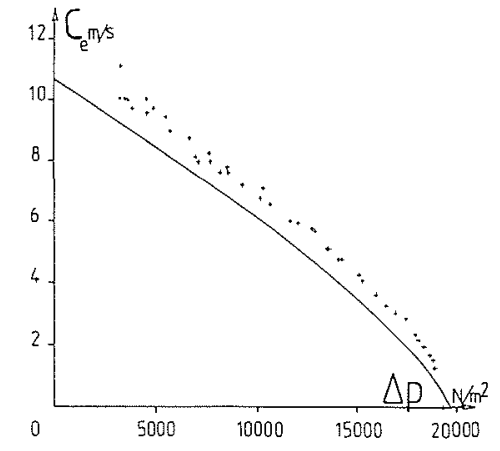

Figure 8 - Vitesse de propagation des ondes de pression pour $\Delta p>0$ :

- courbe calculée par la formule (11)

+ points expérimentaux

\section{Pressions transmurales négatives}

Pour $\Delta p>\Delta p_{c}$, la célérité est obtenue par une dérivation numérique des valeurs $S(\Delta p)$ données par le calcul théorique. Pour $\Delta p<2 \Delta p_{c}$, on déduit des relations (10) et (2):

$$
C_{k}^{2}=-\frac{k \theta}{\rho_{e}} \frac{A_{o}}{S_{i o}}\left(-\frac{\Delta p}{k}\right)^{\frac{\theta-1}{\theta}}-\frac{\theta}{\rho_{e}} \Delta p
$$

Les résultats présentés sur la figure 9 confirment la validité du modèle théorique, la célérité a une valeur minimale de $60 \mathrm{~cm} / \mathrm{s}$ pour $\Delta p_{c}$. La courbe en pointillés donne les valeurs de la célérité dans le tube de caoutchouc seul, rempli d eau et placé dans l'atmosphère, la vitesse de propagation est alors beaucoup plus basse avec un minimum de l'ordre de $20 \mathrm{~cm} / \mathrm{s}$.
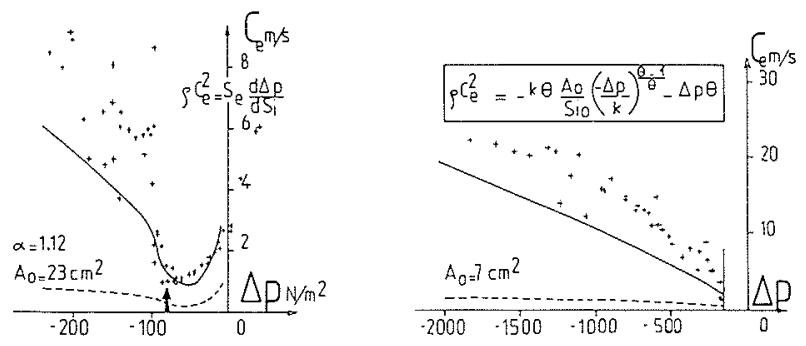

Figure 9 : Vitesse de propagation des ondes de pression pour $\Delta p<0$ :

- courbe calculée à partir de la solution numérique pour $\Delta p>2 \Delta p_{c}$, et d'après la loi de similitude pour $\Delta p<2 \Delta p_{c}$ + points expérimentaux

\section{Etude de l'écoulement}

\section{Dispositif expérimental}

Un tube de latex de longueur $50 \mathrm{~cm}$ et de diamètre $12 \mathrm{~mm}$ est placé dans une enceinte, figure 10. Le passage à travers les parois de l'enceinte est aménagé à travers des membranes élastiques afin de réduire les effets de bouts dus aux encastrements. La prise de pression amont 


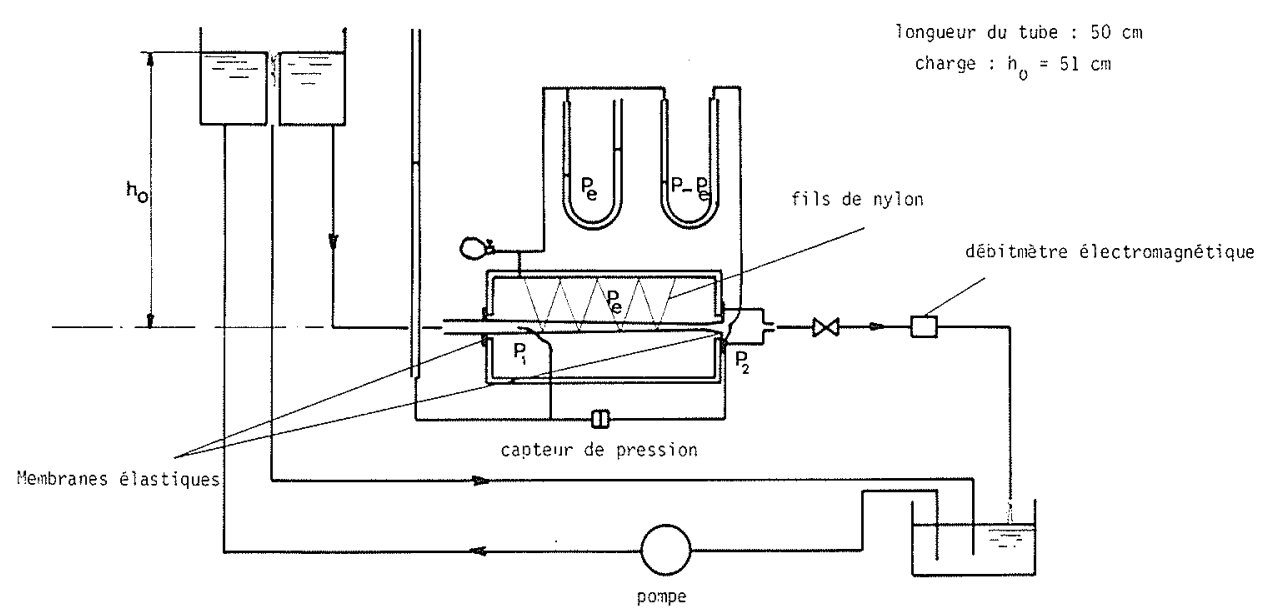

Figure 10 - Dispositif expérimental. Caractéristiques du tube : $a=0,64 \mathrm{~cm}, b=0,49 \mathrm{~cm}, h_{0}=0,31 \mathrm{~mm}, E=1,19 \times 10^{6} \mathrm{~N} / \mathrm{m}^{2}$

est réalisée sur le tube lui-même tandis que la mesure de la pression aval est faite dans un élargissement brusque à la sortie, Bonis et Ribreau [9].

\section{Relations pression-débit}

Pour un tube dont la loi de déformation est $S\left(p_{i}-p_{e}\right)$, le débit $Q$ traversant le tube est fixé par les deux pressions transmurales $p_{1}-p_{e}$ et $p_{2}-p_{e}$. Sur la figure 11 , les courbes expérimentales de $p_{1}-p_{2}$ en fonction de $Q$ sont tracées pour $p_{2}-p_{e}=c^{t e}(-)$, c'est-à-dire à section de sortie constante. Nous en déduisons les caractéristiques pour $p_{1}-p_{e}=c^{\text {te }}(\cdots)$. Certaines conditions d'écoulement étant instables, avec apparition d'oscillations de relaxation, nous avons reporté sur ce graphe les valeurs moyennes $\overline{p_{1}-p_{2}}$.

\section{Courbes $\overline{p_{1}-p_{2}}=f(Q)$ pour $p_{2}-p_{e}$ constante.}

Ces caractéristiques présentent quatre domaines bien distincts que l'on peut caractériser par la "résistance" à l'écoulement définie par la pente $d\left(p_{1}-p_{2}\right) / d Q$

pour $p_{2}-p_{e}>-1 \mathrm{~cm} \mathrm{H} \mathrm{H}_{2} \mathrm{O}$, le tube est ouvert sur toute sa longueur, la résistance à l'écoulement est faible

- $\operatorname{pour} p_{2}-p_{e}<-5 \mathrm{~cm} \mathrm{H}_{2} \mathrm{O}$

- si $p_{1}<p_{c}$, le tube est pincé sur toute sa longueur et l'écoulement se produit dans les deux lobes. La résistance est grande et augmente à mesure que $p_{2}-p_{e}$ diminue;

- si $p_{c}<p_{1}<p_{e}$, on observe une zone de transition non linéaire due aux variations importantes de la section amont dans ce domaine de pression. Le tube s'ouvre de l'amont vers l'aval d'autant plus loin que le débit est élevé;

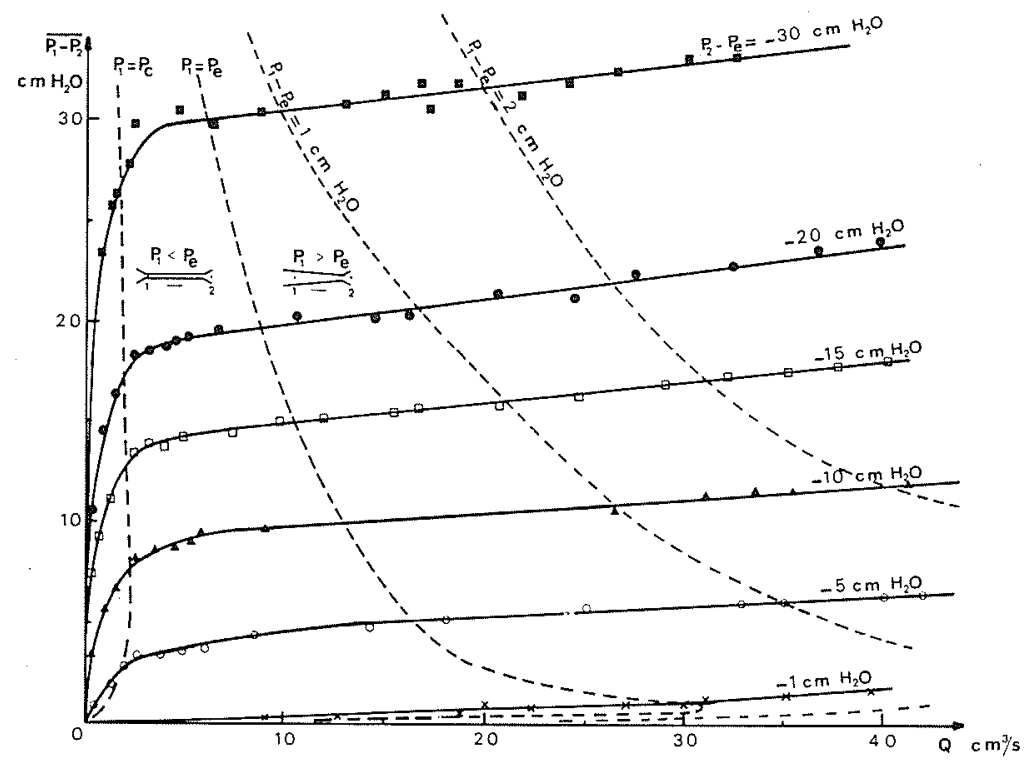

Figure 11 - Relations pression-débit $\left(\Delta p_{c}=-1,6 \mathrm{~cm} \mathrm{H} \mathrm{H}_{2} \mathrm{O}\right)$ :

$-p_{2}-p_{e}=$ constante

-.- $p_{1}-p_{e}=$ constante 
si $p_{1}>p_{e}$, la forme du tube n'évolue pas beaucoup ; il est ouvert sur presque toute sa longueur, sauf à un col situé à l'extrêmité aval. $\mathrm{La}$ résistance est faible et indépendante du débit. De plus elle semble varier peu avec la valeur de $p_{2}-p_{e}$.

\section{Courbes $\overline{p_{1}-p_{2}}=f^{\prime}(Q)$ pour $p_{1}-p_{e}$ constante}

Si $\Delta p_{2}>\Delta p_{c}$ (donc $\Delta p_{1}>\Delta p_{c}$ ), le tube est ouvert sur toute sa longueur, et $Q$ augmente avec $p_{1}-p_{2}$. Tandis que si $\Delta p_{2}<\Delta p_{c}$, l'aval se ferme à mesure que $p_{2}-p_{e}$ diminue (donc que $p_{1}-p_{2}$ augmente) et $Q$ tend vers zéro avec $S_{2}$.

La "résistance négative" dès les faibles valeurs de $p_{1}-p_{2}$ joue un grand rôle dans le retour veineux. En effet, la grande résistance du lit capillaire limite le débit dans les veines qui, de par leur structure, s'adaptent aux conditions aux limites de l'écoulement de manière à rendre les conditions amont indépendantes de l'aval.

Les tubes collabables apparaissent ainsi pouvoir jouer un rôle autorégulateur et s'opposer éventuellement à la cavitation qui pourrait naître d'une forte diminution de la pression aval.

\section{Stabilité de l'écoulement}

Si le tube est alimenté par un réservoir de charge $h_{0}$ constante, la pression motrice en chaque point $M$ à l'intérieur du tube est :

$$
p_{g_{o}}=\rho g h_{o}=p_{g_{M}}+\rho \frac{U_{M^{2}}}{2}+p_{f}
$$

$P_{f}:$ perte de pression par frottements

avec $p_{g_{M}}=p_{e}+\Delta p_{M}$ :

$$
\begin{aligned}
p_{g_{0}}-p_{e}-p_{f}=\frac{\rho U_{M}^{2}}{2}+ & \Delta p_{M}= \\
& \frac{\rho}{2} \frac{Q^{2}}{S^{2}\left(\Delta p_{M}\right)}+\Delta p_{M}=F\left(\Delta p_{M}\right)
\end{aligned}
$$

Si l'on exprime la dérivée $F(\Delta p)$ en fonction de la vitesse de propagation $C(\Delta p)$ :

$$
F(\Delta p)=1-\frac{Q^{2}}{S^{2} C^{2}}=1-\frac{Q^{2}}{Q_{c_{M}}^{2}} \text { avec } C^{2}=\frac{S}{\frac{\rho d S}{d\left(\Delta p_{M}\right)}}
$$

$Q_{c_{M}}$ : débit critique au point $M$.

La figure 12 montre le graphe de la fonction $F$ pour des débits $Q$ constants. Les conditions critiques, tracées en pointillés, délimitent deux domaines dont la discussion a été faite par Comolet [10]. Brièvement:

$F>o:$ l'écoulement est subcritique et stable

$F<o$ : l'écoulement est supercritique. La stabilité dépend alors du couplage entre le comportement

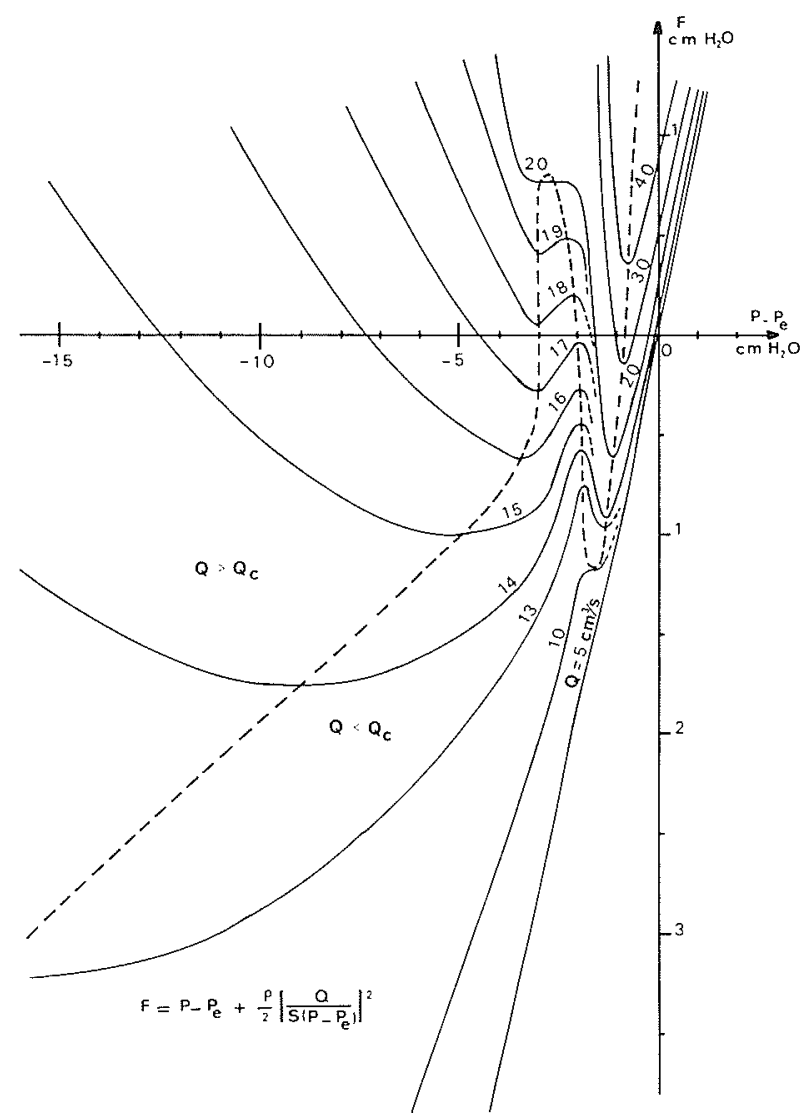

Figure 12 - Graphe de la fonction $F(\Delta p)$ à débit $Q$ constant calculé d'après les valeurs expérimentales de $S(\Delta p)$. La courbe en pointillés correspond au régime critique $(d F / d \Delta p=0)$.

élastique du tube et les propriétés hydrodynamiques de l'écoulement (vitesse et perte de charge).

A partir d'un certain débit, le tube présente des oscillations spontanées de forte amplitude, dont le mécanisme n'a pas encore été clairement élucidé. Nous avons noté sur la figure 13, l'amplitude des fluctuations de pression en chaque point et, afin de voir comment les conditions critiques pourraient être corrélées avec l'apparition de ces oscillations, nous avons reporté en pointillés les conditions critiques calculées pour la pression $p_{2}-p_{e}$.

Si $p_{2}-p_{e}=-5 \mathrm{~cm} \mathrm{H} \mathrm{H}_{2} \mathrm{O}$ et $p_{2}-p_{e}=-10 \mathrm{~cm} \mathrm{H}_{2} \mathrm{O}$, nous avons observé un mouvement de forte amplitude de l'extrémité aval qui s'ouvre et se ferme alternativement. Ces fortes fluctuations de pression (jusqu'à une amplitude de $25 \mathrm{~cm} \mathrm{H}_{2} \mathrm{O}$ ) se produisent dans le domaine délimité en trait mixte sur le graphe. Si pour $p_{2}-p_{e}=$ $-5 \mathrm{~cm} \mathrm{H}_{2} \mathrm{O}$, les instabilités apparaissent exactement aux conditions critiques, pour les pressions aval plus basses elles n'apparaissent que plus tard lorsque l'écoulement est largement supercritique. La fréquence des oscillations augmente avec le débit.

- Pour $p_{2}-p_{e}=-15 \mathrm{~cm} \mathrm{H}_{2} \mathrm{O}$, on n'observe plus que de légères fluctuations près de la sortie.

- Pour $p_{2}-p_{e}=-20 \mathrm{~cm} \mathrm{H}_{2} \mathrm{O}$, il n'y a aucun mouvement visible du tube.

Ces résultats expérimentaux confirment que l'écoulement subcritique est toujours stable. Lorsque l'écou- 


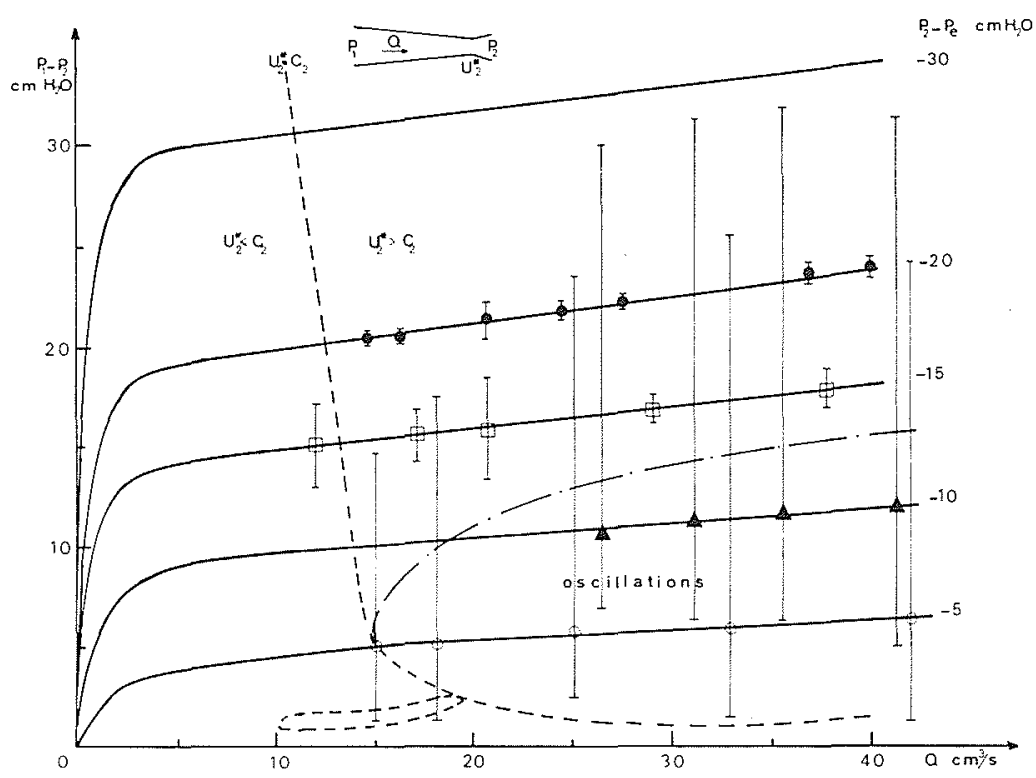

Figure 13 - Relations pression-đébit et amplitude des fluctuations de pression :

... Régime critique calculé pour $p_{2}-p_{e}$

-.- Domaine où se produisent des oscillations de relaxation

Pour $p_{2}-p_{e}<-15 \mathrm{~cm} \mathrm{H}_{2} \mathrm{O}$, les fluctuations de pressions ne sont plus périodiques.

lement devient supercritique, des oscillations de relaxation peuvent apparaître, dont la fréquence dépend des propriétés géométriques et mécaniques de la paroi, mais également de la valeur du débit et des conditions d'encastrement à la sortie. A mesure que le débit augmente, la striction élastique se déplace vers l'aval en se raccourcissant. Cette augmentation de la courbure au col crée une tension longitudinale dans le tube, et ainsi une force de rappel élastique supplémentaire dont l'influence sur la déformation devient prédominante à mesure que $p_{2}-p_{e}$ diminue. Dans ces conditions les valeurs calculées de la vitesse de propagation, qui supposent la conduite de section uniforme, perdent toute signification. La configuration prise par le tube en aval accroit la valeur de la célérité au col et retarde donc l'apparition des instabilités.

\section{Limitation du débit}

La théorie unidimensionnelle de l'écoulement, Oates [11], Dawson et Elliot [12], Shapiro [13], montre que le débit est maximum lorsque la vitesse du fluide est égale à la célérité des ondes de pression au col. Ce phénomène de limitation du débit à sa valeur critique est l'analogue du blocage des tuyères en dynamique des gaz, ou de l'écoulement sur un seuil dans un canal découvert.

Si l'on compare, sur les figures 11 et 13 , les courbes $p_{1}-p_{e}=$ constante et la courbe $u_{2}=C_{2}$ donnant les conditions critiques à l'aval, on peut remarquer que pour la courbe $p_{1}-p_{e}=0$, le débit est bien maximum lorsque l'écoulement est critique. La courbe $p_{1}-p_{e}=$ $1 \mathrm{~cm} \mathrm{H} \mathrm{H}_{2} \mathrm{O}$, bien qu'incomplète, laisse prévoir le mếme résultat en accord avec la théorie unidimensionnelle de l'écoulement.

\section{Conclusion}

Ces quelques résultats expérimentaux mettent en évidence le comportèment caractéristique des tubes flexibles lorsqu'ils sont soumis à des pressions transmurales $\Delta p$ positives ou négatives. Les grandes variations de l'aire intérieure du tube pour les faibles valeurs négatives de $\Delta p$, donc la valeur élevée de $d S / d(\Delta p)$, font que la célérité des ondes de pression a une valeur minimale lorsque les parois opposées du tube viennent en contact. Les relations pression-débit à travers un segment de tube collabable sont gouvernées par la forte non linéarité de la loi de déformation $S(\Delta p)$, et ont des propriétés très différenciées selon la valeur des pressions transmurales amont et aval. Parmi les phénomènes évoqués, certains requièrent des études expérimentales complémentaires, notamment pour prouver que la limitation de débit se produit bien quand les conditions critiques sont atteintes, et pour expliciter le mécanisme des oscillations de relaxation. Ne disposant d'aucune information expérimentale concernant la perte de charge, étant donnés les ordres de grandeur de pression mis en jeu, aucun calcul suffisamment précis ne peut actuellement être envisagé. Des efforts croissants sont actuellement foumis tant dans nos laboratoires qu'à l'étranger pour acquérir les résultats expérimentaux susceptibles d'élucider entre autres ces points particuliers.

\section{Remerciements}

Une partie de cette étude a été financée par l'INSERM, contrat $\mathrm{n}^{\circ}$ 75-1-181-5. Nous remercions M. le Professeur Comolet et M.Y. Jaffrin pour leurs critiques et suggestions au cours de ce travail. 


\section{Références bibliographiques}

[1] BRECHER - History of venous research, IEE Trans, on Biomed, Eng., 1969, Vol BME 16, n 4, 236-247.

[2] HOLT - The collapse factor in the measurement of venous pressure, Am. J. Physiol., 1941, Vol. 134, 292299.

[3] GRIFFITHS - Hydrodynamics of male micturition - I : theory of steady flow through elastic-walled tubes, Med. and Biol. Eng., 1977, Vol. 9, p. 581-588.

[4] KRESCH et NOORDERGRAAF - Cross-sectionnal shape of collapsible tubes, Biophys. J., 1972, Vol. 12, 274-294.

[5] SCHERER, KAMM, SHAPIRO - External pneumatic compression for the prevention of deep venous thrombosis, Proc. of the San Diego Biomed. Symp., 1975, Vol $14,403-407$

[6] TRELOAR - The Physics of rubber elasticity, 1958, Clarendon Press

[7] RIBREAU et BONIS - Propagation et écoulement dans les tubes collabables. Contribution à l'étude des vaisseaux sanguins, 2ème Congrès de la Société de Biomécanique sept. 77, Liège.

[8] TAYLOR et GERRARD - Pressure-radius relationships for elastic tubes and their applications to arteries, $\mathrm{Med}$. and Biol. Eng. and Comp., 1977, Vol. 15, 11-17.

[9] BONIS et RIBREAU - Pressure-flow relationships in collapsible tubes, Euromech 92, Dynamique Cardiovas. culaire et Pulmonaire, Sept. 1977, Ed. M.Y. Jaffrin, Série des Colloques INSERM. Vol. 71.

[10] COMOLET - Sur la nature et la stabilité de l'écoulement unidimensionnel dans une conduite deformable élastiquement, Euromech 92, Dynamique Cardiovasculaire et Pulmonaire, Sept. 1977. Ed. M.Y. Jaffrin, Série des Collo ques INSERM, Vol. 71.

[11] OATES - Fluid flow in soft-wailed tubes, Med. and Biol. Eng., 1975, Vol. 13, 773-784.

[12] DAWSON et ELLIOT - Wave-speed limitation on expiratory flow - a unifying concept, J. Appl. Physiol Respirat. Environ. Exercise Physiol, 1977, Vol. 43, 498. 515.

[13] SHAPIRO - Steady flow in collapsible tubes, $J$. Biomech. Eng., Aug. 1977, 126-147.

\section{Discussion}

Président : M.J. HINGLAIS

A l'ouverture de la Séance, M. REVOL, Secrétaire général de la S.H.F., présente à l'assemblée les excuses du Président CAZENAVE retenu par ailleurs.

Il m'a laissé, poursuit-il, la lourde charge de vous présenter le Président: le Professeur HINGLAIS de l'équipe d'études chirurgicales à l'Hôpital Broussais, un vieil ami de la Société Hydrotechnique de France.

Il y a une dizaine d'années $M$. HINGLAIS avait déjà participé à nos travaux de biohydraulique à propos de problèmes de circulation sanguine.

Il a bien voulu accepter de présider la séance de cet aprèsmidi : nous l'en remercions et je lui passe immédiatement la parole.

Avant de donner la parole au premier conférencier, le Président propose à l'assistance de recenser les principales "spécialités" dont elle est composée.

A son appel se manifestent successivement les médecins, les physiciens-hydrauliciens et les biomécaniciens.

Le Président en conclut qu'il y a une majorité de biomécaniciens, quelques physiciens fondamentalistes et quelques médecins.

Il donne la parole à M. BONIS.

Après l'exposé de celui-ci, le Président remercie M. BONIS et attire, en ces termes, l'attention sur tout l'in térêt que présente son exposé.

Le domaine des tuyaux collabables est encore extrêmement mal étudié, alors qu'il couvre tout le système veineux systématique, tout le système pulmonaire, soit plus de la moitié de notre circulation sanguine. Ils obéissent à des mécanismes que l'on comprend encore extrêmement mal.

Au contraire, nos études dans le domaine artériel sont beaucoup plus poussées. Les mécanismes y sont beaucoup plus faciles à comprendre. Les méthodes de mesures y sont plus aisées.
La circulation veineuse a pourtant une importance considérable.

Déjà au sortir de l'organe, on a affaire à de très fins vaisseaux qui doivent le drainer en dépit d'une très faible pression, sous peine de voir survenir un œdème.

Il y a aussi cet extraordinaire mouvement de sang, dû aux forces aux alentours de la paroi veineuse, qui, lorsqu'on est debout ramène la quasi-totalité du débit cardiaque depuis les pieds jusqu'au cœur, dans une cavité - l'oreillette - où la pression n'est pas supérieure à $5 \mathrm{~mm}$ de mercure.

L'absence de travaux vraiment poussés sur les tuyaux collabables est dûe à la difficulté de ces travaux, et je remercie beaucoup M. BONIS de s'être attaché au problème. Je trouve son étude excellente.

J'aimerais connaître l'avis des médecins "médicinants" sur l'exposé qu'ils viennent d'entendre.

Le Docteur SEBBAN répond en observant que l'exposé de M. BONIS fait appel à des notions scientifiques qui ne sont pas toujours très familières aux médecins.

Dans la mesure où ils rejoignent les travaux sur le retour veineux, poursuit-il, limportance des travaux sur les tuyaux collabables est absolument indiscutable. Il conviendrait notamment d'examiner si ils permettraient d'affiner ce qu'en physiologie nous appelons le schéma de Guyton, un schéma essentiel pour la compréhension physiologique du système cardiovasculaire.

Une question de M. GERAL - physiologiste à Toulouse relative à la circulation pulmonaire est l'occasion pour M. BONIS, d'insister sur le caractère encore très schématique des problèmes qu'il a traités. "Les phénomènes, précise-t-il "sont encore si mal connus et surtout si mal quantifiés qu'on ne peut pas se lancer dans des applications physiologiques en raison de leur complexité accrue. C'est encore un peu tôt".

Sur une nouvelle question de M. GERAL, M. BONIS confirme le caractère essentiellement non-linéaire des relations pression-débit qu'il a pu dégager.

Le Président observe que la partie artérielle de la circulation pulmonaire est sans doute - pour plusieurs raisons - le siège de variations de pression à la paroi assez notables. Il demande à M. BONIS si les courbes qu'il a tracées peuvent s'y appliquer. 
M. BONIS indique en réponse qu'il ne sait pas dans quelle mesure les capillaires pulmonaires peuvent s'écraser.

En réponse à des questions de $\mathrm{M}$. ODDOU sur la validité de la loi : $\mathrm{C}^{2}=\mathrm{S} / \rho \delta \Delta \mathrm{P} / \delta \Delta \mathrm{S}$ dans les faibles sections et sur la nature des hypothèses limitatives concernant la forme et les variations relatives de la section, M. BONIS indique qu'il s'agit de la célérité d'une onde infinitésimale de pression et que l'application du théorème des quantités de mouvement n'implique aucune hypothèse particulière à cet égard.

Une autre question de $\mathrm{M}$. ODDOU amène M. BONIS à préciser, comme suit, les conditions de mesure des vitesses de propagation. Lorsque le tube intérieur est gonflé, donc avec un espace annulaire réduit, la mesure est faite entre deux capteurs de pression. On envoie une impulsion dans la conduite et on mesure l'intervalle de temps séparant l'émission et le passage de l'onde.

Lorsque le tube est collabé, très écrasé, les choses deviennent plus délicates parce que l'amplitude des ondes de pression mises en jeu est très faible et que les capteurs ne l'enregistrent plus.

On a mesuré la vitesse avec des fils chauds, qui voient très bien passer les vitesses parce qu'ils ont une sensibilité très granđe. Les résultats obtenus étaient cohérents.

M. BONNIN observe que pour les tuyaux mécaniques, les Hydrauliciens ont l'habitude de calculer les célérités d'ondes en négligeant l'inertie des parois parce que les déplacements sont très faibles. Il demande si, pour des conduites collabables ayant une grande déformation, elle est encore négligeable ?

$\mathrm{Au}$ cours du bref débat que suscite cette question, M. COMOLET rappelle, comme suit, les conditions d'application de la formule de Moens Korteweg donnant la célérité des ondes de pression. Cette formule suppose des mouvements du fluide uniquement longitudinaux et fait donc abstraction de toute composante radiale de la vitesse. Elle suppose donc que les déplacements radiaux de la paroi sont négligeables. On ne tient pas compte des variations radiales de la quantité de mouyement de la paroi ; et par conséquent on ne fait pas état de la masse volumique de la paroi.

En cas de grandes variations radiales de la paroi, il n'en serait pas de même et la formule de Moens-Korteweg ne serait plus applicable.

M. BONIS indique avoir observé qu'à un pincement léger sur le tube correspondait une certaine valeur de la vitesse de propagation et qu'une impulsion de forte amplitude se propageait à une vitesse supérieure.

Diverses questions de M. QUEMADA, de M. COLLINS et de $M$. KOPP amènent $M$. BONIS à préciser la nature des régimes instables observés du type oscillant spontané et le rôle à son avis négligeable de la viscoélasticité vis-à-vis des grandes déformations non-linéaires de la section. Malgré des déplacements importants, les déformations de la paroi restent d'ordre infinitésimal.

Les oscillations de grande amplitude observées par $M$ BONIS comporteraient une dent de scie, avec un petit retour a des fréquences de l'ordre de 2 à $3 \mathrm{~Hz}$.

M. GERAL puis M. QUEMADA reviennent au cas de la microcirculation pulmonaire. M. QUEMADA note que des instabilités semblent apparaître dans le milieu alvéolaire.

L'existence de modes instables, précise-t-il, est certaine lorsque la viscoélasticité existe.

Il demande à M. BONIS si la prise en compte éventuelle de tels effets sur son modèle ne ferait pas apparaître de nouveaux domaines d'instabilité, comme dans tous systèmes dissipatifs.

M. BONIS observe que l'amplitude du phénomène est trop grande pour que la viscoélasticité la fasse disparaître ; tout au plus peut-elle diminuer cette amplitude.

A son avis, d'autre part, les capillaires pulmonaires ne présentent pas d'élargissement de section assez brusque pour provoquer un tel phénomène.

Enfin on peut attribuer la naissance des oscillations à une instabilité statique ou dynamique du tube, lorsque les conditions critiques sont atteintes; ce qui n'était pas le cas.

En conclusion, M. BONIS estime que si la théorie unidimensionnelle n'apparaît pas comme la seule valable, elle n'est pas exclue pour autant : il y a deux explications possibles.

M. QUEMADA remarque que le modèle de M. BONIS suppose une élasticité linéaire lorsque la section devient très petite. Il demande si les effets de courbure importants, qui apparaissent lorsque le tube est collabé, n'imposent pas une limite assez sévère à la théorie linéaire et si cette limite est atteinte ou non dans les expériences.

M. BONIS pense également qu'il y a une limite mais qu'à la limite la section tend vers zéro.

M. REMENIERAS observe que pour le calcul des coups de bélier dans les conduites industrielles, on néglige la déformation longitudinale de celles-ci. Cette simplification est-elle encore acceptable dans le cas des tubes collabables étudiés par les auteurs?

M. BONIS précise que, dans le cas où le tube est sollicité au gonflement, le coefficient d'extension longitudinal intervient explicitement sous forme du terme $\alpha$.

Lorsque le tube est écrasé, sous une surpression extérieure, il y a une sollicitation longitudinale car le tube est de longueur constante; ces effets ne sont pas pris en compte par le calcul qui suppose le tube de longueur infinie. 